\title{
Penile herpes zoster: an unusual location for a common disease
}

\begin{abstract}
Herpes zoster is a common dermatological condition which affects up to $20 \%$ of the population, most frequently involving the thoracic and facial dermatomes with sacral lesions occurring rarely and only a few reported cases of penile shingles. Case report: We report two cases of unusual penile clinical presentations of varicella zoster virus infection in immunocompetent men. The patients presented with grouped clusters of vesicles and erythema on the left side of penile shaft and posterior aspect of the left thigh and buttock, involving S2-S4 dermatomes. The lesions resolved quickly upon administration of oral antiviral therapy. Conclusion: Penile herpes zoster should not be overlooked in patients with unilateral vesicular rash.
\end{abstract}

Keywords: herpes zoster; penis neuralgia; postherpetic.

Two immunocompetent patients referred to Venereology Department of Institute for Skin Diseases, complaining on vesicular penile rash.

A 30-year-old man presented with rash in the groin which appeared five days before referral. The patient denied any constitutional symptoms as well as prodromal symptoms of pain or burning but did report itching in the affected area. Physical examination showed herpetic clusters of hemorrhagic vesicles and pustules on an erythematous base distributed on the left side of penile shaft and posterior aspect of the left thigh (Figures 1 and 2). Hematological, biochemical and urine analysis were within normal limits. A 7-day course of acyclovir (800 mg five times a day) was initiated. The course of the disease remained uncomplicated and the lesions healed without sequelae.

The second patient, a 72-year-old man presented with vesicular rash in the groin that developed approximately one week before he sought medical attention. The lesions were accompanied with severe pain along the S2-S4 dermatomes without constitutional symptoms of fever, headache or malaise. Physical examination showed clusters of blisters on an erythematous base mostly filled with hemorrhagic fluid, distributed on the penile shaft and left buttock (Figures 3 and 4), without regional lymphadenopathy. He denied any

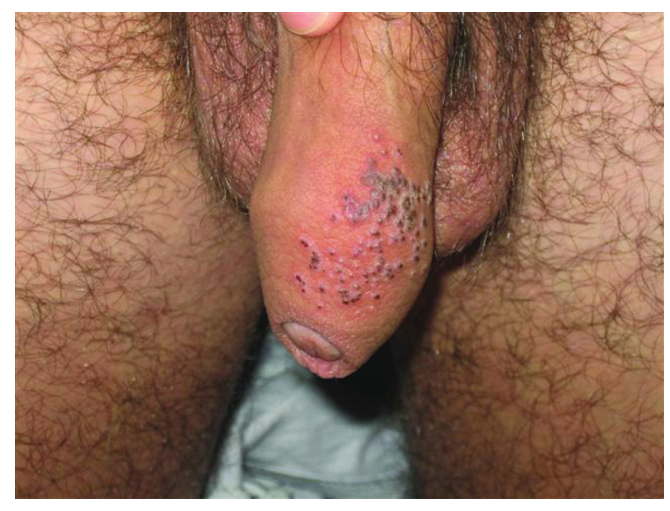

Figure 1: Clusters of hemorrhagic vesicles on the left side of penile shaft.

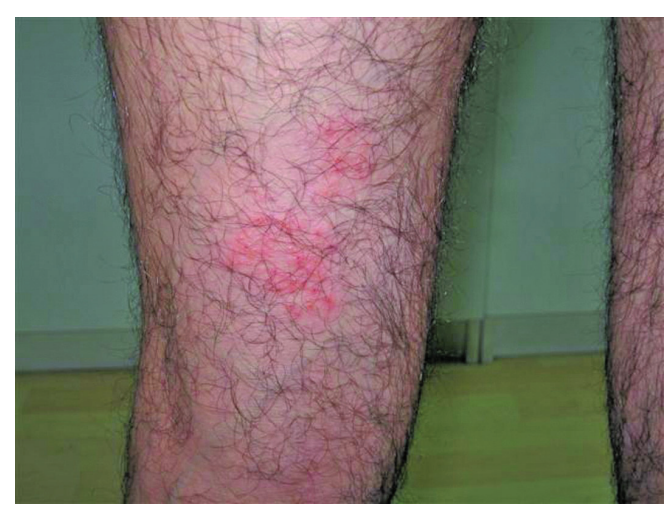

Figure 2: Discrete vesiculopapules on erythematous base in the posterior aspect of the left thigh.
Authors

Milan Bjekic ${ }^{1}$

Milica Markovic ${ }^{2}$

Sandra Sipetic ${ }^{3}$

${ }^{1} \mathrm{MD}, \mathrm{PhD}$; Head of

Department of Venereal

Diseases, City Institute for

Skin and Venereal Diseases,

Belgrade, Serbia

${ }^{2} \mathrm{MD}$, Practicing

Dermatologist, City

Institute for Skin and

Venereal Diseases,

Belgrade, Serbia

${ }^{3} \mathrm{MD}, \mathrm{PhD}$; Institute of

Epidemiology, School

of Medicine, Belgrade

University, Belgrade, Serbia

Submitted on: 04/29/2011 Approved on: 05/28/2011

Correspondence to:

Milica Markovic

Dzorza Vasingtona 17,

11000, Belgrade

Serbia, Europe

mipopovi@eunet.rs

Financial Support: This work was supported by the Ministry for Science and Technology of Serbia, through Contract No. 175042 (2011-2014).

We declare no conflict of interest.

(ㄷ2011 Elsevier Editora Ltda. All rights reserved. 


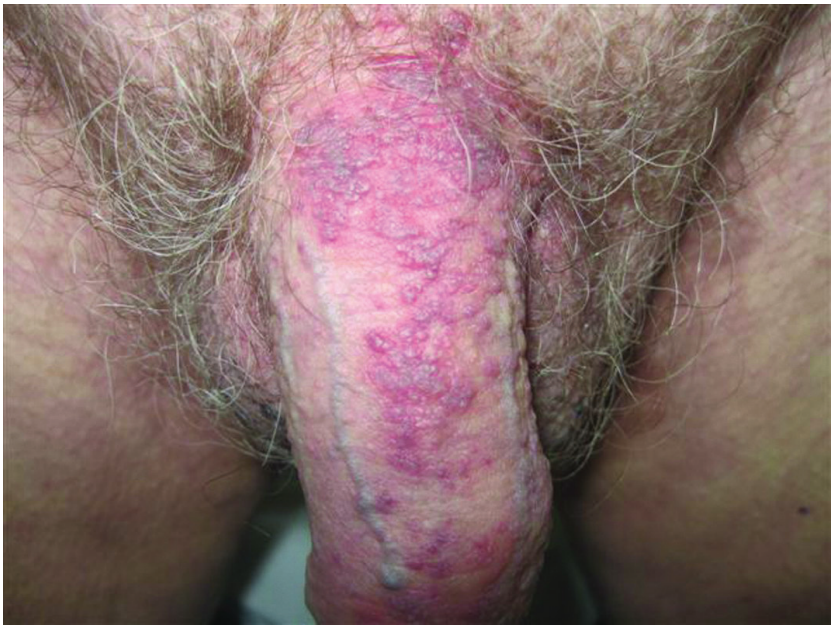

Figure 3: Haemorrhagic vesicles and blisters on penile shaft.

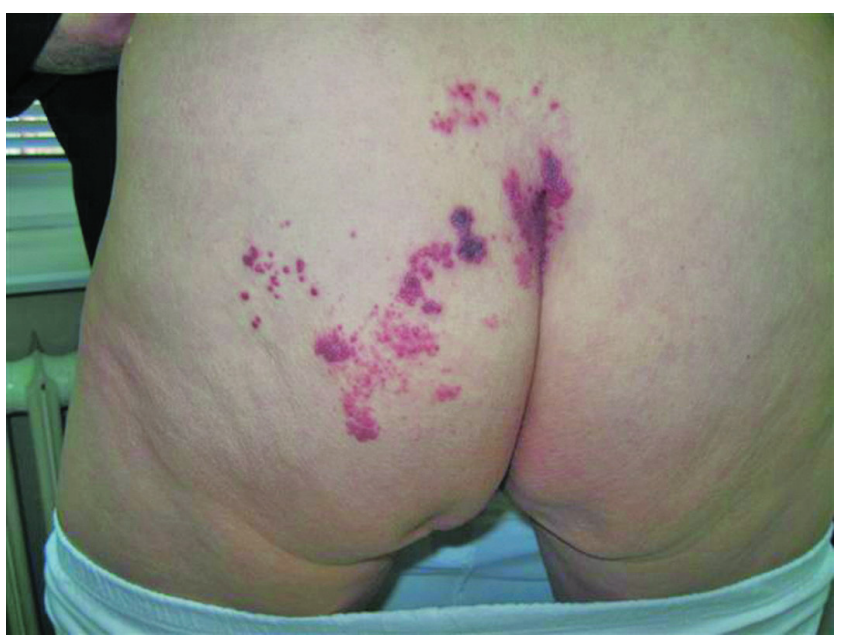

Figure 4: Zosteriform arrangement of herpetic vesicles on the left buttock.

urinary symptoms. Medical history revealed type II diabetes. Routine investigations including liver and renal function tests were normal. The patient was treated with acyclovir $800 \mathrm{mg}$ five times daily for one week. Skin lesions resolved after three weeks, but the disease was complicated with post-herpetic neuralgia. Amitriptyline, a tricyclic antidepressant (25 mg three times a day), was initiated and provided moderate pain relief.

Herpes zoster predominantly affects the trunk in up to $50 \%-60 \%$ of cases, followed by the head region (10\%-20\%) with sacral dermatomes involved in only up to $5 \%$ of cases. ${ }^{1}$ Penile zoster is neither commonly seen by dermatologists nor reported in dermatological journals., ${ }^{2,3}$ The diagnosis of herpes zoster is made clinically; however laboratory confirmation is necessary only in atypical inconclusive clinical cases. Both patients appeared with penile vesicular rash involving S2-S4 dermatomes. Post-herpetic neuralgia is the most frequently reported complication, and risk factors include older age, more severe acute pain and greater rash severity. ${ }^{4}$ Our patient with post-herpetic neuralgia was of older age and had type II diabetes, compared to an otherwise healthy younger patient with acute penile zoster without pain.

Bladder dysfunction and urinary retention have been reported in patients with sacral herpes zoster $^{5}$ but were absent in the cases presented herein. Penile herpes zoster should not be overlooked in patients with unilateral vesicular rash.

\section{REFERENCES}

1. Glynn C, Crockford G, Gavaghan D, et al. Epidemiology of shingles. J R Soc Med. 1991; 84:184.

2. Spray A, Glaser DA. Herpes zoster of the penis: an unusual location for a common eruption. J Am Acad Dermatol. 2002; 47:S177-9.

3. Chandrashekar L, Kumar DA, Mohan TD. Penile zoster with urethritis. Indian J Sex Transm Dis. 2004; 25:81-3.

4. Whitley RJ, Shukle S, Crooks RJ. The identification of risk factors associated with persistant pain following herpes zoster. J Infect Dis. 1998; 178:S71-5.

5. McElhaney JE. Herpes zoster: a common disease that can have a devastating impact on patients quality of life. Expert Rev Vaccines. 2010; 9(Suppl 3):27-30. 